\title{
Bumblebee Flight in Heavy Turbulence
}

\author{
T. Engels, ${ }^{1,2}$ D. Kolomenskiy, ${ }^{3}$ K. Schneider, ${ }^{1}$ F.-O. Lehmann, ${ }^{4}$ and J. Sesterhenn ${ }^{2}$ \\ ${ }^{1}$ M2P2-CNRS \& Aix-Marseille Université, 38 rue Joliot-Curie, 13451 Marseille cedex 20 France \\ ${ }^{2}$ ISTA, Technische Universität Berlin, Müller-Breslau-Strasse 12, 10623 Berlin, Germany \\ ${ }^{3}$ Graduate School of Engineering, Chiba University, 1-33 Yayoi-Cho, Inage-Ku, Chiba-Shi, Chiba 263-8522, Japan \\ ${ }^{4}$ Department of Animal Physiology, University of Rostock, Albert-Einstein-Strasse 3, 18059 Rostock, Germany \\ (Received 2 September 2015; revised manuscript received 24 November 2015; published 15 January 2016)
}

High-resolution numerical simulations of a tethered model bumblebee in forward flight are performed superimposing homogeneous isotropic turbulent fluctuations to the uniform inflow. Despite tremendous variation in turbulence intensity, between $17 \%$ and $99 \%$ with respect to the mean flow, we do not find significant changes in cycle-averaged aerodynamic forces, moments, or flight power when averaged over realizations, compared to laminar inflow conditions. The variance of aerodynamic measures, however, significantly increases with increasing turbulence intensity, which may explain flight instabilities observed in freely flying bees.

DOI: 10.1103/PhysRevLett.116.028103

Insect flight currently receives considerable attention from both biologists and engineers. This growing interest is fostered by the recent trend in miniaturization of unmanned air vehicles that naturally incites reconsidering flapping flight as a bioinspired alternative to fixed-wing and rotary flight. For all small flyers it is challenging to fly outdoors in an unsteady environment, and it is essential to know how insects face that challenge.

Field studies show variations of insect behavior with changing weather conditions, including the atmospheric turbulence [1]. Earlier laboratory research on aerodynamics of insect flight assumed quiescent air, and only some more recent experiments focused on the effect of different kinds of unsteady flows. The behavior of orchid bees flying freely in a turbulent air jet has been studied in [2]. The authors found that turbulent flow conditions have a destabilizing effect on the body, most severe about the animal's roll axis. In response to this flow, bees try to compensate the induced moments by an extension of their hind legs, increasing the roll moment of inertia. Interaction of bumblebees with wake turbulence has also been considered in [3]. These experiments were performed in a von Kármán-type wake behind cylinders. The bees displayed large rolling motions, pronounced lateral accelerations, and a reduction in their upstream flight speed. In [4] a comparative study on the sensitivity of honeybees and stalk-eye flies to localized wind gusts was performed. The study found that bees and stalkeye flies respond differently to aerial perturbations, either causing roll instabilities in bees or significant yaw rotations in stalk-eye flies. In [5] feeding flights of hawk moths in vortex streets past vertical cylinders were analyzed. Depending on the distance of the animal from the cylinder and cylinder size, destabilizing effects on yaw and roll and a reduction in the animal's maximum flight speed have been observed. Kinematic responses to large helical coherent structures were also found in hawk moths flying in a vortex chamber [6]. A study on the energetic significance of kinematic changes in hummingbird feeding flights further demonstrated a substantial increase in the metabolic rate during flight in turbulent flows, compared to flight in undisturbed laminar inflow [7,8]. All studies reported significant changes in the behavior of insects when they fly in turbulent flows and incite the question if, and how, the efficiency of flapping wings changes. It is critical to understand whether the aerodynamic challenge insects face when flying through turbulence is due to the elevated power requirements and reduced force production, or rather limited capacity of flight controls. Experiments with freely flying animals involve complex, sensory-dependent changes in wing kinematics and wing-wake interaction. To isolate specific effects of turbulence on aerodynamic mechanisms and power expenditures in flight, direct numerical simulations are well-suited tools. However, to determine statistical moments of the forces and torques acting on the insect, a sufficient number of flow realizations needs to be computed, owing to the generic randomness of turbulence.

In this Letter, we present the first direct numerical simulations of insect flight in fully developed turbulence, using a model bumblebee based on [9]. We address the question of how turbulence alters forces, moments, and power expenditures in flapping flight. Since bumblebees are all-weather foragers, they encounter a particularly large variety of natural flow conditions [3]. We consider our model bumblebee in forward flight at $2.5 \mathrm{~m} / \mathrm{s}$, flapping its rigid wings [Fig. 1(a)] at a Reynolds number of 2042. To conduct the simulation, we designed a "numerical wind tunnel" and placed the animal in a $6 R \times 4 R \times 4 R$ large, virtual, rectangular box, where $R=13.2 \mathrm{~mm}$ is the wing length. The computational domain is discretized with 680 million grid points and the incompressible threedimensional Navier-Stokes equations are solved by direct numerical simulation using the software described in [10]. An imposed mean inflow velocity accounts for the forward flight speed of the tethered insect, with superimposed 


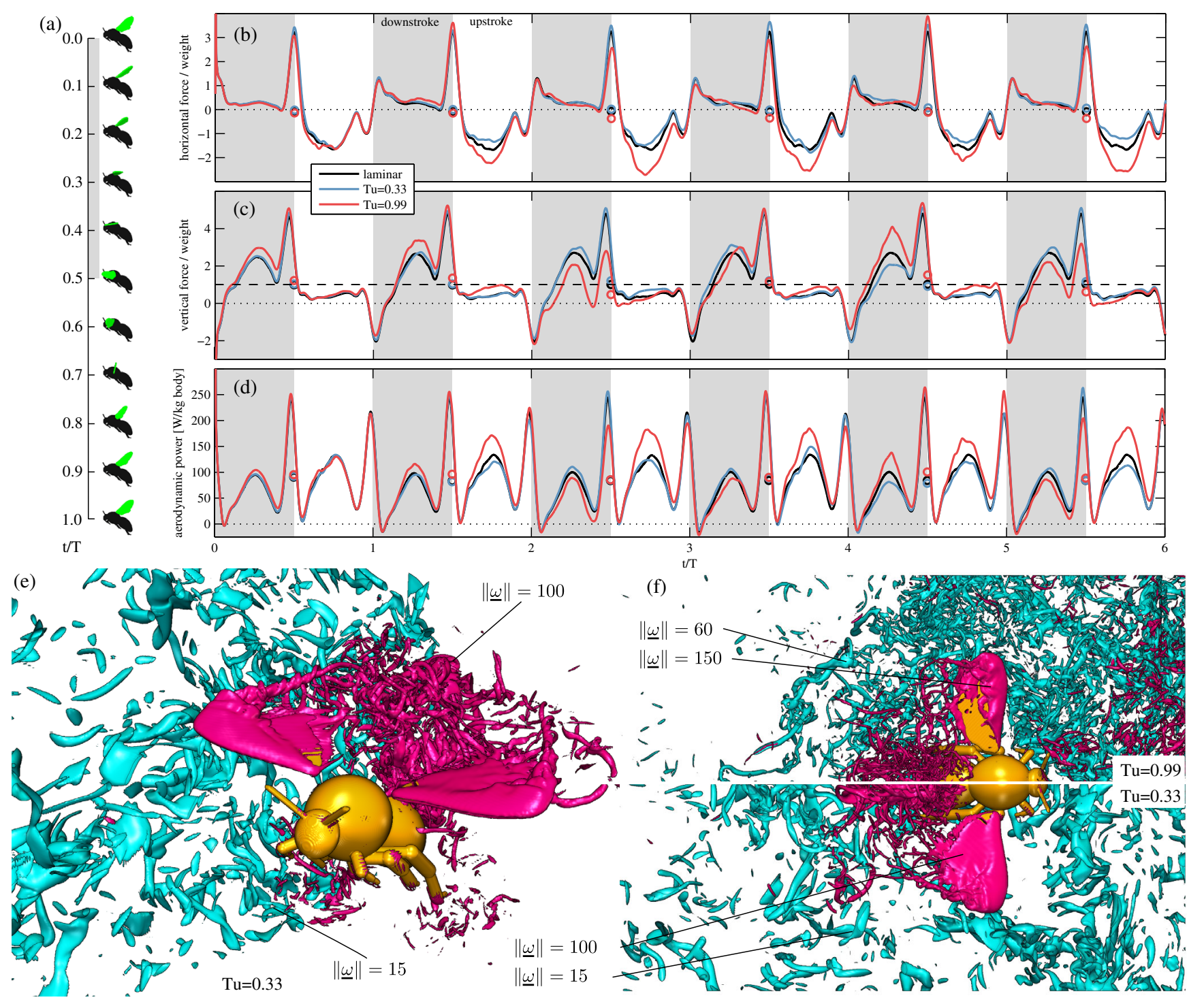

FIG. 1. Bumblebee in turbulent flow. (a) Visualization of the prescribed wingbeat, where $T$ is period time. (b)-(d) Time evolution of horizontal (b) and vertical (c) force, and aerodynamic power (d) under laminar, moderately turbulent (Tu $=0.33$ ) and highly turbulent $(\mathrm{Tu}=0.99)$ conditions. Circular markers represent cycle-averaged values. (e)-(f) Flow visualization by means of isosurfaces of normalized vorticity magnitude $\|\underline{\omega}\|$. (e) Perspective view for a realization with $\mathrm{Tu}=0.33$. The purple and blue isosurfaces visualize stronger and weaker vortices, respectively, and weaker vortices are shown only for $3.7 R \leq y \leq 4 R$. (f) Top view, with the upper half showing flow at elevated turbulent $(\mathrm{Tu}=0.99)$, and the lower half at moderate turbulent $(\mathrm{Tu}=0.33)$ intensity. Weaker vortices, i.e., smaller values of $\|\underline{\omega}\|$, are shown only for $0 \leq z \leq 0.3 R$.

velocity fluctuations in the turbulent cases. Since the actual properties of these aerial perturbations depend on a large number of parameters, we model them by homogeneous isotropic turbulence (HIT) [11,12]. This is a reasonable assumption for the small turbulent scales relevant to insects. In addition, HIT is a well-established type of turbulence which reduces the set of parameters to the turbulent Reynolds number $R_{\lambda}$. Insect flight can thus be studied from laminar to fully developed turbulent flow conditions, yielding time series of aerodynamic measures [Figs. 1(b)-1(d)], as well as the flow data [Figs. 1(e) and 1(f)]. Further details on the model and the simulations can be found in the Supplemental Material [13].
First, we focus on the wake pattern generated by the insect in laminar inflow. This case serves as reference for the turbulence simulations and provides quantitative data on vortical flow generated by the flapping wings. Figures 1(b)-1(d) show how body weight-normalized lift and thrust, and body mass specific aerodynamic power vary throughout the flapping cycles. Force and power peak during the stroke reversals, as observed in [22]. The cycleaveraged flight forces obtained from this simulation are summarized in Table I. The data show that the bumblebee model produces lift that matches the weight to within $2 \%$, but $8 \%$ more thrust than required to compensate for free stream velocity. These slight discrepancies likely result 


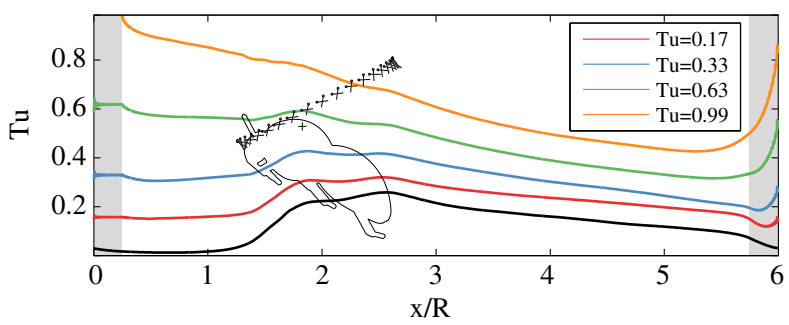

FIG. 2. Slab averaged turbulence intensity as a function of the axial coordinate, with the insect drawn to scale for orientation. The black line is the laminar case. The gray shaded areas mark regions where the inflow and outflow is imposed.

from the uncertainty of the input parameters. The aerodynamic power required to actuate the wings is $84 \mathrm{~W} / \mathrm{kg}$ body mass. This is larger than the value reported in [23] $(56 \mathrm{~W} / \mathrm{kg}$ body mass), which may be explained by the differences in the wing kinematics and the aerodynamic models employed. Mean moments about the three rotational body axes do not significantly differ from zero suggesting a torque balanced force production. The turbulence intensity, $\mathrm{Tu}=u^{\prime} / u_{\infty}$, is the root mean square (rms) of velocity fluctuations normalized to flight velocity. Figure 2 presents slab-averaged turbulence intensity $\langle\mathrm{Tu}\rangle=\int_{y_{0}-1.3 R}^{y_{0}+1.3 R} \int_{z_{0}-1.3 R}^{z_{0}+1.3 R} \mathrm{Tu}(x, y, z) d y d z /(2.6 R)^{2}$ as $\mathrm{a}$ function of the downstream distance. The black line corresponds to the laminar case. The subdomain used for averaging is centered around the insect $\left(y_{0}=2 R\right.$, $z_{0}=2 R$ ). Complementary $3 \mathrm{D}$ visualizations of the wake can be found in the Supplemental Material [13]. The data show that the bumblebee model generates relative intensities of $25 \%$ at the wings and approximately $16 \%$ at five wing lengths downstream distance. This finding indicates that relevant turbulence intensities are larger than $16 \%$, which is well above the inflow turbulence considered in investigations concerning airfoils, typically below $1 \%$ [24].

Second, we study the model insect in turbulent inflow considering four different turbulence intensities, with turbulent Reynolds numbers $R_{\lambda}=\lambda u^{\prime} / \nu$ ranging from 90 to 228 . Here $u^{\prime}$ is the rms velocity, $\lambda$ the Taylor microscale, varying between $0.25 R$ and $0.1 R$, and $\nu$ is the kinematic viscosity of air. The properties of the inflow data are summarized in Table II. For all tested intensities, the Kolmogorov length scale of small, dissipating eddies, $\ell_{\eta}$, is significantly smaller than the wing length. The length scale of energy carrying structures, the integral scale $\Lambda$, is similar to the wing length. The latter is expected to maximize the impact of turbulence on the insect, while the former suggests that all vortices generated by the insect interact nonlinearly with inflow perturbations. To obtain statistically reliable mean values and variances, we perform $N_{R}$ simulations. Figures 1(e) and 1(f) illustrate the flow under turbulent inflow conditions for $\mathrm{Tu}=0.33$ and 0.99 relative intensity. It shows that weak turbulence is associated with relatively coarse flow structures in the inflow. In contrast, flow patterns near the wings are similar in size and intensity to the structures present in the inflow at strong turbulence. The streamwise slab-averaged turbulence intensity (Fig. 2) is increased by the flapping wings for Tu equal to 0.17 and 0.33 , while it remains constant or is decreased for Tu equal to 0.63 and 0.99 . The lower two values can thus be referred to as mild turbulence.

The considered range of turbulent Reynolds numbers covers the flow regime that a bee typically encounters in its natural habitats. Bumblebees have been reported to fly at wind speeds of $8 \mathrm{~m} / \mathrm{s}$ [25]. At this speed, habitats with cylindrical trees of about $10 \mathrm{~cm}$ in diameter yield turbulent Reynolds numbers in the range considered here. Figures 1(b)-1(d) show that lift, thrust, and power of single simulation runs at turbulent conditions differ from the measures obtained for the laminar case. However, the generic features of the data, i.e., the location of peaks and valleys, are similar under all tested flow conditions; see Sec. IA of the Supplemental Material [13]. Wingbeataveraged and ensemble-averaged data including statistics are shown in Table I. The mean values demonstrate only negligible differences between turbulent and laminar flow conditions and even at the strongest turbulent perturbation, the bumblebee model generates mean aerodynamic forces close to those derived in unperturbed inflow, at virtually the same energetic cost. This aerodynamic robustness of insect wings is in striking contrast to the properties of streamlined airfoils that are highly sensitive to the laminar-turbulent transition [24]. Figure 3 shows the vortical structure at the wing at $t / T=0.3$, represented by the $\|\underline{\omega}\|=100$ isosurface of normalized vorticity $\underline{\omega}=(\nabla \times \underline{u}) / f$. The laminar case is a snapshot of the flow field, while turbulent data are phase averaged over $N_{w}$ independent strokes for each value of Tu (see Table II). Although turbulence alters the shape and size of the wing's tip vortex, the leading edge vortex remains visible in phase-averaged flow fields even at maximum inflow turbulence intensity.

TABLE I. Aerodynamic forces, power, and moments obtained in the numerical experiments. Forces are normalized by the weight $m g$, moments by $m g R$, power is given in $\mathrm{W} / \mathrm{kg}$ body mass. Values are given by mean value $\bar{x}, 95 \%$ confidence interval $\delta_{95}$, and standard deviation $\sigma$ in the form $\bar{x}^{ \pm \delta_{95}} \pm \sigma$.

\begin{tabular}{lccccccc}
\hline \hline Tu & Forward force $F_{h}$ & Vertical force $F_{v}$ & Aerodynamic power $P_{\text {aero }}$ & Moment $M_{x}$ (roll) & Moment $M_{y}$ (pitch) & Moment $M_{z}$ (yaw) \\
\hline 0 & $-0.08^{ \pm 0.0} \pm 0.0$ & $1.02^{ \pm 0.0} \pm 0.0$ & $84.05^{ \pm 0.0} \pm 0.0$ & $0.00^{ \pm 0.0} \pm 0.0$ & $0.01^{ \pm 0.0} \pm 0.0$ & $0.00^{ \pm 0.0} \pm 0.0$ \\
0.17 & $-0.10^{ \pm 0.04} \pm 0.08$ & $1.04^{ \pm 0.09} \pm 0.18$ & $83.72^{ \pm 1.77} \pm 3.61$ & $-0.01^{ \pm 0.01} \pm 0.03$ & $+0.00^{ \pm 0.02} \pm 0.03$ & $-0.01^{ \pm 0.02} \pm 0.03$ \\
0.33 & $-0.06^{ \pm 0.09} \pm 0.18$ & $1.10^{ \pm 0.10} \pm 0.21$ & $85.02^{ \pm 2.03} \pm 4.14$ & $-0.01^{ \pm 0.04} \pm 0.08$ & $-0.01^{ \pm 0.03} \pm 0.06$ & $+0.04^{ \pm 0.02} \pm 0.05$ \\
0.63 & $+0.02^{ \pm 0.10} \pm 0.29$ & $1.04^{ \pm 0.13} \pm 0.40$ & $83.32^{ \pm 3.13} \pm 9.57$ & $-0.02^{ \pm 0.04} \pm 0.12$ & $+0.02^{ \pm 0.04} \pm 0.12$ & $+0.07^{ \pm 0.04} \pm 0.13$ \\
0.99 & $-0.10^{ \pm 0.07} \pm 0.37$ & $1.01^{ \pm 0.10} \pm 0.54$ & $85.44^{ \pm 1.98} \pm 10.47$ & $+0.01^{ \pm 0.04} \pm 0.19$ & $-0.04^{ \pm 0.03} \pm 0.13$ & $-0.03^{ \pm 0.04} \pm 0.21$ \\
\hline \hline
\end{tabular}




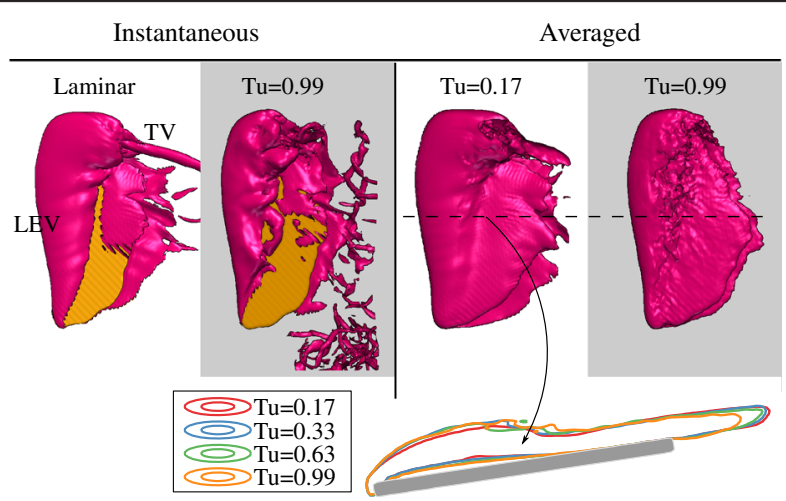

FIG. 3. Top: Isosurface of normalized absolute vorticity, $\|\underline{\omega}\|=100$, in the vicinity of the right wing at $t / T=0.3$. Snapshots of instantaneous vorticity distribution during laminar and turbulent inflow are shown on the left. Phase- and ensembleaveraged vorticity from 16 and 108 wingbeats and at $\mathrm{Tu}=0.17$ and $\mathrm{Tu}=0.99$, respectively, is shown on the right. Bottom: averaged $\|\underline{\omega}\|=50$ isolines at midspan for all values of Tu. The leading edge vortex persists on average even under the strongest inflow perturbations.

Previous studies highlighted that turbulent flows may destabilize the body posture of an insect [2]. Roll, in particular, is prone to instability because the roll moment of inertia is approximately four times smaller than about the other axes. Our results in Table I show that mean aerodynamic moments about yaw, pitch, and roll axes do not change with increasing turbulence. However, we observe characteristic changes in moment fluctuation. Assuming that during perturbation the insect begins to rotate from rest at time $t_{0}$, we may approximate the final angular roll velocity from

$$
\Omega_{\mathrm{roll}}\left(t_{0}+\tau\right)=\frac{1}{I_{\text {roll }}} \int_{t_{0}}^{t_{0}+\tau} M_{\text {roll }}(t) d t,
$$

with $M_{\text {roll }}$ the roll moment, $I_{\text {roll }}$ the roll moment of inertia with respect to the body $x$ axis, and $\tau$ the response delay (see below) [26]. The maximum turbulence-induced roll velocity that a freely flying bumblebee encounters depends on the reaction time of the animal in response to changes in body posture. Many insects compensate for posture perturbations by asymmetrically changing their wing stroke. Previous studies on freely flying honeybees reported response delays of approximately $20 \mathrm{~ms}$ or 4.5 stroke cycles, suggesting the use of ocellar pathways for body stability reflexes in this species [4].

TABLE II. Parameters of inflow turbulence used in simulations. The Kolmogorov length scale $\ell_{\eta}$, the Taylor micro $\lambda$, and the integral scale $\Lambda$ are normalized by the wing length $R$. For each value of Tu, a number of $N_{R}$ realizations have been performed, yielding in total $N_{w}$ statistically independent wingbeats.

\begin{tabular}{lccccrr}
\hline \hline$R_{\lambda}$ & $\mathrm{Tu}$ & $\ell_{\eta}$ & $\lambda$ & $\Lambda$ & $N_{R}$ & $N_{w}$ \\
\hline 90.5 & 0.17 & 0.013 & 0.246 & 0.772 & 4 & 16 \\
130.1 & 0.33 & 0.008 & 0.179 & 0.782 & 4 & 16 \\
177.7 & 0.63 & 0.005 & 0.129 & 0.759 & 9 & 36 \\
227.9 & 0.99 & 0.004 & 0.105 & 0.759 & 27 & 108 \\
\hline \hline
\end{tabular}

To predict the maximum delay that allows a bumblebee to recover from turbulence-induced roll, the response delay in Eq. (1) is set to $\tau=2,3$, and 4 stroke periods. Figure 4 shows how the rms final roll velocity increases under these conditions with increasing turbulence intensity. Previous behavioral measurements provide an estimate of the body angular velocity from which insects can restabilize in free flight. Figure 4 thus predicts that bumblebees recover from turbulence-induced roll motions up to $\mathrm{Tu}=0.63$ assuming response delays between two and four stroke periods. In contrast, posture recovery at $\mathrm{Tu}=0.99$ requires reduced reaction times of not more than two cycle periods, implying that bumblebees cannot achieve stable flight at $\mathrm{Tu}=0.99$. This conclusion is consistent with experimental observations of orchid bees crashing in strongly turbulent flows when flying freely [2].

In conclusion, high-resolution numerical experiments of a bumblebee in perturbed forward flight highlighted several unexpected results with respect to alterations in aerodynamic forces, flight stability, and aerodynamic power expenditures. The simulations imply that even the strongest background turbulence does not vitally harm the structure and efficacy of the lift-enhancing leading edge vortex and thus averaged forces and moments are almost identical compared to laminar flow conditions. Turbulent inflow conditions are thus of little significance for the overall flight performance of an animal in tethered flight. However, these fluctuations cause temporal transient effects. Thus, in a freely flying insect in which the body may rotate, absolute angular velocities about yaw, pitch, and roll axes might reach elevated values, which in turn would require decreasing reaction response delays for body stabilization with increasing turbulence. Owing to its small moment of inertia, roll is especially prone to turbulence-induced fluctuations.

An important consequence of body roll is the deflection of the wingbeat-averaged resultant aerodynamic force from the vertical direction. Thus, at large roll angles, the animal must increase the magnitude of this force so that its vertical component can support the weight of the insect, at the cost of

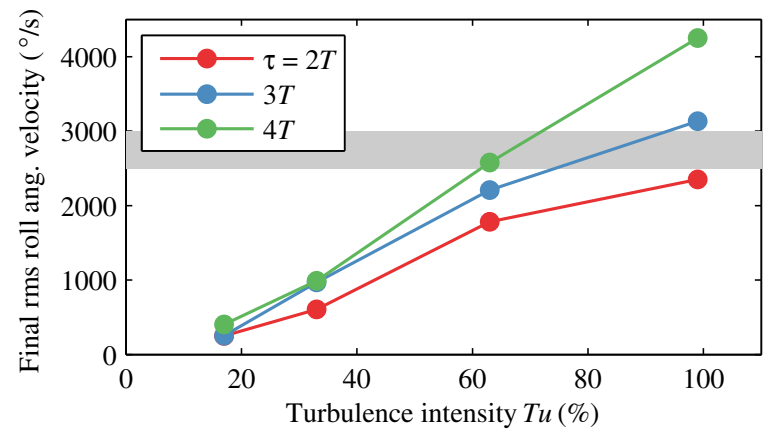

FIG. 4. rms value of the final roll angular velocity $\Omega_{\text {roll }}$ versus the inflow turbulence intensity, calculated over all flow realizations. The colors correspond to different response delay times $\tau$. The gray shaded area represents the limit of sensor saturation, estimated from the behavioral measurements available for honeybees [4] and fruit flies [27]. 
larger aerodynamic power. Incidentally, it has been reported that hummingbirds increase the wingbeat frequency and amplitude [8]. The finding that an increase in inflow turbulence intensity has no significant effect on power expenditures for tethered flight is surprising and significant with respect to flight endurance and migration of insects. Since it has been suggested that flight of insects is limited by power rather than force production [28], any biological and physical mechanisms that help an insect to limit its wing and body drag-dependent power expenditures is of great value and may increase the animal's biological fitness.

As the leading edge vortex is a common feature in many flapping flyers, we expect these conclusions to generally hold also at different flight speeds and for other species as well. This is also suggested by our results in the Supplemental Material [13] obtained with different morphology, kinematics, and Reynolds number.

We thank H. Liu and M. Maeda for their advice on the kinematic modeling of flapping wings, and S. Ravi for many valuable comments on the manuscript. This work was granted access to the HPC resources of Aix-Marseille Université financed by the project Equip@Meso (ANR-10-EQPX-29-01) and IDRIS under Project No. i20152a1664. T. E., K. S., and J. S. acknowledge financial support from DFH-UFA, and D. K. from the JSPS postdoctoral fellowship P15061.

[1] S. M. Swartz, K. S. Breuer, and D. J. Willis, Aeromechanics in aeroecology: Flight biology in the aerosphere, Integr. Comp. Biol. 48, 85 (2008).

[2] S. A. Combes and R. Dudley, Turbulence-driven instabilities limit insect flight performance, Proc. Natl. Acad. Sci. U.S.A. 106, 9105 (2009).

[3] S. Ravi, J. Crall, A. Fisher, and S. A. Combes, Rolling with the flow: Bumblebees flying in unsteady wakes, J. Exp. Biol. 216, 4299 (2013).

[4] J. T. Vance, I. Faruque, and J. S. Humbert, Kinematic strategies for mitigating gust perturbations in insects, Bioinspiration Biomimetics 8, 016004 (2013).

[5] V. M. Ortega-Jimenez, J. S. M. Greeter, R. Mittal, and T. L. Hedrick, Hawkmoth flight stability in turbulent vortex streets, J. Exp. Biol. 216, 4567 (2013).

[6] V. M. Ortega-Jimenez, R. Mittal, and T. L. Hedrick, Hawkmoth flight performance in tornado-like whirlwind vortices, Bioinspiration Biomimetics 9, 025003 (2014).

[7] V. M. Ortega-Jimenez, N. Sapir, M. Wolf, E. A. Variano, and R. Dudley, Into turbulent air: Size-dependent effects of von Kármán vortex streets on hummingbird flight kinematics and energetics, Proc. Biol. Sci. 281, 20140180 (2014).

[8] S. Ravi, J. D. Crall, L. McNeilly, S. F. Gagliardi, A. A. Biewener, and S. A. Combes, Hummingbird flight stability and control in freestream turbulent winds, J. Exp. Biol. 218, 1444 (2015).

[9] R. Dudley and C. P. Ellington, Mechanics of forward flight in bumblebees I. Kinematics and morphology, J. Exp. Biol. 148, 19 (1990).
[10] T. Engels, D. Kolomenskiy, K. Schneider, and J. Sesterhenn, FluSI: A novel parallel simulation tool for flapping insect flight using a Fourier method with volume penalization, arXiv:1506.06513; FluSI: A novel parallel simulation tool for flapping insect flight using a Fourier method with volume penalization, SIAM J. Sci. Comput. (to be published).

[11] R. S. Rogallo, Numerical Experiments in Homogeneous Turbulence (NASA Technical Memorandum, NASA, Hampton, VA, 1981), Vol. 81315, p. 1.

[12] Y. Kaneda, T. Ishihara, M. Yokokawa, K. Itakura, and A. Uno, Energy dissipation rate and energy spectrum in high resolution direct numerical simulations of turbulence in a periodic box, Phys. Fluids 15, L21 (2003).

[13] See Supplemental Material at http://link.aps.org/ supplemental/10.1103/PhysRevLett.116.028103, which includes Refs. [14-21], for [supporting results, materials and methods].

[14] P. Angot, C. Bruneau, and P. Fabrie, A penalization method to take into account obstacles in incompressible viscous flows, Numer. Math. 81, 497 (1999).

[15] T. Engels, D. Kolomenskiy, K. Schneider, and J. Sesterhenn, Numerical simulation of fluid-structure interaction with the volume penalization method, J. Comput. Phys. 281,96(2015).

[16] S. N. Fry, R. Sayaman, and M. H. Dickinson, The aerodynamics of hovering flight in Drosophila, J. Exp. Biol. 208, 2303 (2005).

[17] D. Kolomenskiy, H. K. Moffatt, M. Farge, and K. Schneider, Two- and three-dimensional numerical simulations of the clapfling-sweep of hovering insects, J. Fluids Struct. 27, 784(2011).

[18] M. Maeda and H. Liu, Ground effect in fruit fly hovering: A three-dimensional computational study, J. Biomech. Sci. Eng. 8, 344 (2013).

[19] D. Pekurovsky, P3DFFT: A framework for parallel computations of Fourier transforms in three dimensions, SIAM J. Sci. Comput. 34, C192 (2012).

[20] N. Xu and M. Sun, Lateral dynamic flight stability of a model bumblebee in hovering and forward flight, J. Theor. Biol. 319, 102 (2013).

[21] "University of Minnesota Insect Collection," http:// insectcollection.umn.edu, accessed 2014-05-14.

[22] M. H. Dickinson, F.-O. Lehmann, and S. P. Sane, Wing rotation and the aerodynamic basis of insect flight, Science 284, 1954 (1999).

[23] R. Dudley and C. P. Ellington, Mechanics of forward flight in bumblebees: II. Quasi-steady lift and power requirements, J. Exp. Biol. 148, 53 (1990).

[24] T. Mueller, L. Pohlen, P. Conigliaro, and B. Jansen, The influence of Free-stream disturbances on low Reynolds number airfoil experiments, Exp. Fluids 1, 3 (1983).

[25] T. J. Wolf, C. P. Ellington, and I. S. Begley, Foraging costs in bumblebees: Field conditions cause large individual differences, Insectes Sociaux 46, 291 (1999).

[26] S. N. Fry, R. Sayaman, and M. H. Dickinson, The aerodynamics of free-flight maneuvers in Drosophila, Science $\mathbf{3 0 0}$, 495 (2003).

[27] L. Ristroph, A. J. Bergou, G. Ristroph, K. Coumes, G. J. Berman, J. Guckenheimer, Z. J. Wang, and I. Cohen, Discovering the flight autostabilizer of fruit flies by inducing aerial stumbles, Proc. Natl. Acad. Sci. U.S.A. 107, 4820 (2010).

[28] C. Ellington, The novel aerodynamics of insect flight: applications to micro-air vehicles, J. Exp. Biol. 202, 3439 (1999). 